\title{
UNDER THE MICROSCOPE
}

Jose M. Carnate Jr., MD

Marvin C. Masalunga, MD²

'Department of Pathology

College of Medicine

University of the Philippines Manila

${ }^{2}$ Department of Laboratories

Philippine General Hospital

University of the Philippines Manila

\section{Intracapsular Carcinoma ex Pleomorphic Adenoma}

This is the case of a 37-year-old female patient presenting with a 10-year history of a gradually enlarging right infra-auricular mass. A parotidectomy was performed. The surgical pathology specimen consisted of an $18 \mathrm{~cm}$ diameter encapsulated nodular mass with a homogenous, cream-tan solid surface. Microscopic section showed an encapsulated neoplasm with abundant chondromyxoid stroma and tubular epithelial elements characteristic of a pleomorphic adenoma. (Figure 1) Randomly scattered within the tumor were foci of haphazard and complex glands. (Figure 2) These glands exhibited nuclear pleomorphism, luminal necrosis and mitoses compatible with an adenocarcinomatous proliferation. (Figure 3) Based on these features, the case was signed out as an intracapsular carcinoma ex pleomorphic adenoma.

Carcinoma ex pleomorphic adenoma is a carcinoma arising from a pre-existing pleomorphic adenoma. The antecedent benign tumor may either be a long-standing one often with a history measured in decades, or characterized by a protracted history of excisions and multiple recurrences. ${ }^{1,2}$ The carcinoma on the other hand is either epithelial or myoepithelial in derivation. By morphologic sub-type the most commonly reported carcinoma arising in a pleomorphic adenoma is a salivary duct carcinoma or an adenocarcinoma that is not otherwise specified (NOS). ${ }^{1,3}$ Residual pleomorphic adenoma tissue is identifiable either in its typical morphology, a chondromyxoid stroma, or a hyalinized sclerotic nodule.'

Aside from the type of carcinoma arising from the pleomorphic adenoma, another parameter that has to be reported is the extent of involvement by the carcinomatous component. A carcinoma that is entirely limited to within the parent tumor that is still enclosed by a complete capsule is termed an "intracapsular" or "non-invasive" carcinoma ex pleomorphic

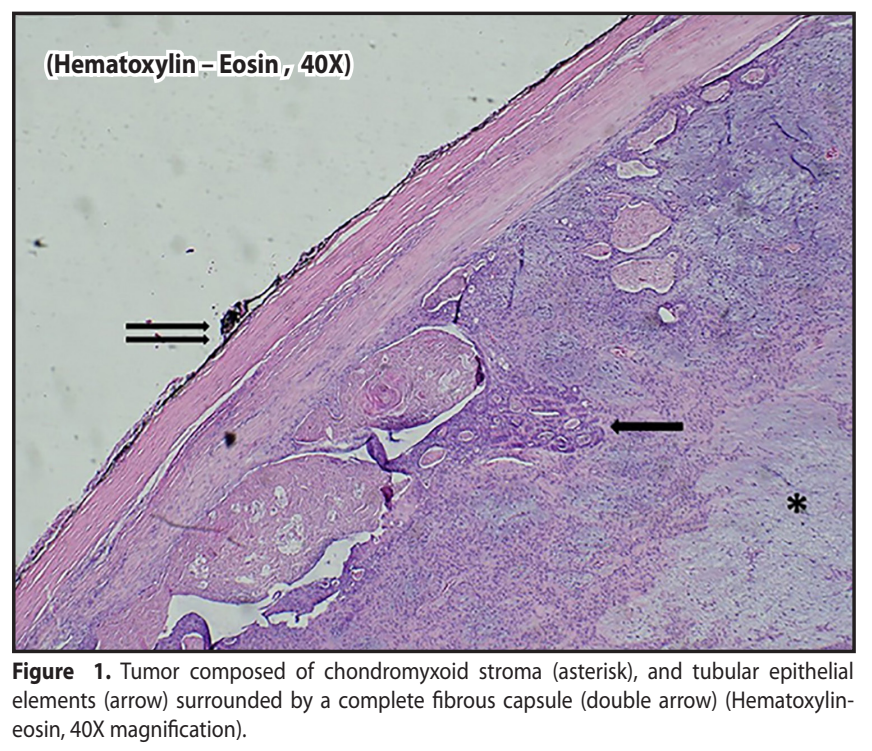




\section{UNDER THE MICROSCOPE}

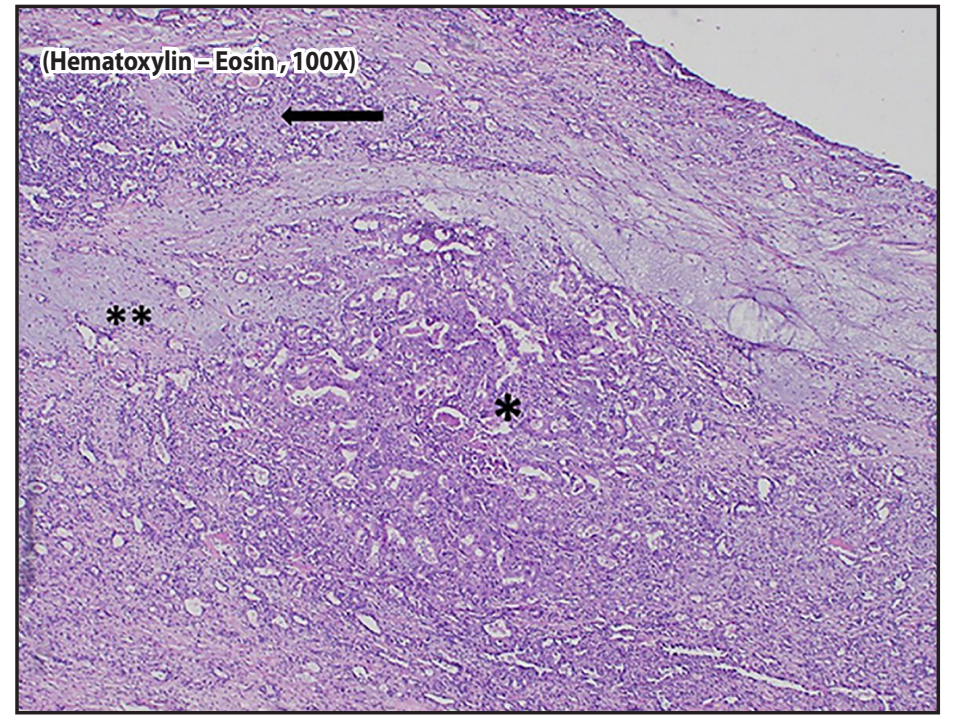

Figure 2. Haphazard and complex glands (asterisk) surrounded by chondromyxoid stroma (double asterisk) and tubular epithelial elements (arrow) (Hematoxylin-eosin, 100X magnification).

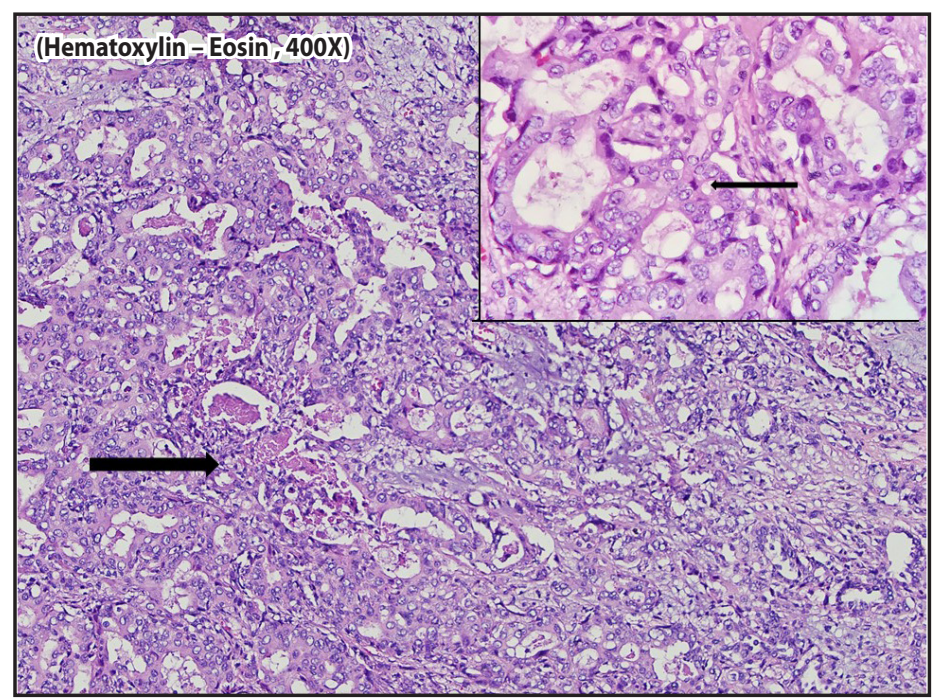

Figure 3. Haphazard and complex glands with luminal necrosis (thick arrow). Inset shows atypical nuclei and a mitosis (thin arrow) (Hematoxylin-eosin, 400X magnification).

adenoma. ${ }^{1,2}$ Once the carcinoma breaches the capsule and infiltrates the surrounding tissue, then it is considered invasive. If the invasion is less than $4-6 \mathrm{~mm}$ beyond the capsular border, the tumor is termed "minimally invasive". Carcinomatous elements that extend beyond this threshold is termed "widely invasive"." This threshold is greater than the previous threshold of $1.5 \mathrm{~mm}$ recommended in an earlier edition of the WHO classification although the present edition does state that this threshold is preliminary and requires further validation. ${ }^{1,2,4}$ It has to be pointed out though that quantifying invasion may not always be possible in tumors that have positive margins, those that are intrinsically unencapsulated such as minor salivary gland tumors, and those with complex multinodular growth patterns such as recurrent pleomorphic adenoma. ${ }^{1}$ This difficulty has to be stated in the report and what conditions preclude quantifying the degree of invasion.

Non-invasive carcinoma ex pleomorphic adenoma has quite a good outcome with very low reported rates of recurrence or regional metastasis. In a review of thirty cases and a report of an additional three cases, only one case showed recurrence or metastasis. ${ }^{3}$ This favorable outcome certainly contrasts with that of the widely invasive type where metastasis is reported to occur in up to $70 \%$ of cases. ${ }^{1}$ Another review of ten cases showed one case developing metastasis, and recommended that non-invasive cases should thus still be followed up closely after primary treatment because regional or distant metastasis can occur. ${ }^{2}$

To the best of our knowledge, there are no published local data on the incidence of early malignant transformation of pleomorphic adenomas in the Filipino population. Hence, we take this opportunity to report this case. Awareness of the entity and prudent liberal sampling of these tumors may help address this gap. 